\title{
Dialogue games for Dishkant's quantum modal logic
}

\author{
VLADIMIR L. VASYUKOV ${ }^{1}$
}

\begin{abstract}
Recently some elaborations were made concerning the game theoretic semantic of $\mathrm{E}_{\aleph_{0}}$ and its extension. In the paper this kind of semantics is developed for Dishkant's quantum modal logic $\mathrm{EQ}$ which is also, in fact, the specific extension of $\mathrm{L}_{\aleph_{0}}$. As a starting point some game theoretic interpretation for the SE system (extending both Łukasiewicz logic $\mathrm{E}_{\aleph_{0}}$ and modal logic S5) was exploited which has been proposed in 2006 by C. Fermüller and R. Kosik. They, in turn, based on ideas already introduced by Robin Giles in the 1970th to obtain a characterization of $\mathrm{E}_{\aleph_{0}}$ in terms of a Lorenzen style dialogue game combined with bets on the results of binary experiments that may show dispersion.
\end{abstract}

Keywords: Łukasiewicz's logic, quantum loigic, dialogue games, risk value

\section{Introduction}

In [4],[5] Robin Giles determines a logic for reasoning about physical theories with dispersive experiments, meaning that repeated trials of the same experiment may yield different results. Giles refers to Lorenzen's dialogue games for intuitionistic and classical logic which systematically reduce arguments involving logically complex assertions to arguments about atomic assertions.

In the issue Robin Giles formally defined a characterization of infinite-valued Lukasiewicz logic in terms of a game that combines dialogue rules for logical connectives with a scheme for betting on results of dispersive experiments for evaluating atomic propositions.

\footnotetext{
${ }^{1}$ This study comprises research findings from the 'Game-theoretical foundations of pragmatics' Project № 12-03-00528 carried out within The Russian Foundation for Humanities Academic Fund Program.
} 
In this connection it is interesting that Herman Dishkant introduced the modal extension of Łukasiewicz's infinite-valued logic which allows to consider physical objects obeying to the rules of quantum mechanics. This suggests to extend Giles' method to Dishkant's logic for obtaining a characterization of that in terms of a dialogue game too. The starting position and conditions in this case would be as follows.

The main idea of H. Dishkant's quantum modal logic ( $\mathrm{EQ})[1]$ is to include Mackey's axioms for probabilities of quantum-mechanical experiments [6] into the calculus of Łukasiewicz's infinite-valued logic $\mathrm{E}_{\aleph_{0}}$ treating probabilities as truth-values. It is done not directly and Mackey's construction plays the role of a leading idea only and resulting calculus is, in essence, a modal extension of Eukasiewicz logic where the last is enriched with the modal symbol $Q$ and four modal inference rules. The proposition $Q A$ expresses such a property which can be observed and the presence of which confirms $A$ (' $A$ is confirmed by observation').

The system $\mathrm{L} Q$ contains four axioms and five rules of inference:

A1. $A \rightarrow(B \rightarrow A)$

A2. $(A \rightarrow B) \rightarrow((B \rightarrow C) \rightarrow(A \rightarrow C))$

A3. $((A \rightarrow B) \rightarrow B) \rightarrow((B \rightarrow A) \rightarrow A)$

$\mathbf{A 4}(\neg A \rightarrow \neg B) \rightarrow(B \rightarrow A)$

B5. $\frac{A, A \rightarrow B}{B}$

B6. $\frac{A}{Q A}$

B7. $\frac{A}{\neg Q \neg A}$

B8. $\frac{A \rightarrow B}{Q A \rightarrow Q B}$ 
B9. $\frac{Q A \rightarrow Q B}{(Q B \rightarrow Q A) \leftrightarrow Q(Q B \supset Q A)}$

where $A \supset B={ }_{\text {def }}(\neg A \rightarrow B) \rightarrow B$.

Semantically Dishkant' system $\mathrm{E} Q$ would be interpreted in the following way. Usually a quantum object is described by a wave function - by a unit vector of a complex Hilbert space $H R$. Let $\Psi$ be the set of all states of an object and besides these states we consider also questions which are described by closed subspaces of $H R$. Each such closed subspace $\hat{p}$ determines a probability $p(\psi)$ of a positive answer to the question for any $\psi \in \Psi$. It is known that this probability is equal to the squared modulus of the projection of $\psi$ on the subspace $\hat{p}$, i.e. $p(\psi)=\left|\psi_{\hat{p}}\right|^{2}$.

Since $\hat{p}_{1} \neq \hat{p}_{2} \Rightarrow p_{1} \neq p_{2}$ then we do not identify the question with the corresponding function of $H R$ but with the corresponding function $p: \Psi \rightarrow[0,1]$ for which there exists such $\hat{p}$ that $p(\psi)=$ $\left|\psi_{\hat{p}}\right|^{2}$. Here $[0,1]$ is the closed segment of real numbers.

Let $\mathcal{P}$ be the set of all questions and for any $p \in \mathcal{P}$ let $\hat{p}$ be the corresponding subspace of $H R$. We call any function $g: \Psi \rightarrow[0,1]$ a generalized question and the set of all generalized questions will be denoted by $\mathcal{S}$. Obviously $\mathcal{P} \subset \mathcal{S}$. The set $\mathcal{S}$ is partially ordered by the relation $\leq$ which is defined by

$$
g \leq h={ }_{\text {def }} \forall \psi(q(\psi) \leq h(\psi)) \text { for any } g, h \in \mathcal{S} .
$$

Now let us fix a function $q: \mathcal{S} \rightarrow \mathcal{P}$ satisfying the conditions

q1. $g \leq h \Rightarrow q(g) \leq q(h)$

q2. $q(p)=p$

for any $g, h \in \mathcal{S} ; p \in \mathcal{P}$. It is easy to see that there is at least such a function $q$ (e.g. one may take $q(g)$ equal to that $p$ for which $\hat{p}$ is the minimal subspace containing all $\psi \in \Psi$ for which $q(\psi)=1$ ).

Any function $I^{D}: W^{0} \rightarrow S$ is an interpretation of $\mathrm{\iota} Q$ if it satisfy the following conditions:

(I) $I^{D}(A \rightarrow B)=\min \left(1,1-I^{D}(A)+I^{D}(B)\right)$; 
(II) $I^{D}(\neg A)=1-I^{D}(A)$;

(III) $I^{D}(Q A)=q\left(I^{D}(A)\right)$

for any $A, B \in W^{0}$, where $W^{0}-$ a set of formulas of $\mathrm{L} Q$. Here 1 : $\Psi \rightarrow\{1\}$, where $\Psi$ is a set of all states of an object. It is obvious that $I^{D}$ may be defined on $V$ (the infinite list of propositional variables) arbitrarily and then extended uniquely on $W^{0}$, if $q$ is fixed.

It seems that under such definition $q$ plays for modal formules the same role as Mackey's function $r$ which assigns to every triple $(A, \alpha, E)$ (where $A$ is an observable, $\alpha$ is a state and $B$ is a Borel subset of the real line) the number $r(A, \alpha, E), 0 \leq r(A, \alpha, E) \leq 1$. So, we can treat $W^{0}$ as the set of observables, $\operatorname{dom}(S)$ as the set of states and $r n g(S)$ as the set of all Borel subsets of the real line.

The following result holds for such an interpretation $I^{D}[1$, p. 152]:

Theorem 1. For any $A \in W^{0}$, if $\vdash A$ then $I^{D}(A)=1$ for any intepretation $I^{D}$.

The weak completeness (semantic correctness) of $\mathrm{\iota} Q$ was proved just relative to the usual quantum propositional logic $Q P L$ (by embedding $Q P L$ in $\mathrm{E} Q$ ). In view of this the problem was formulated to construct semantic model like those of Kripke-Grzegorczyk but for $\mathrm{E} Q$. In [7] such Kripke-type model for $\mathrm{E}_{\aleph_{0}}$ was yielded where an accessibility relation is a ternary one and in [8, p. 67] such model was extended to $\mathrm{\iota} Q$ and the soundness and completeness of $\mathrm{\iota} Q$ in respect to those was proved.

According to [8] the ternary semantic of $\mathrm{\iota} Q$ would be described as follows. E-frame is a quadruple $\left\langle O, K, R,{ }^{*}\right\rangle$ where $K$ is nonempty set of observation points (states), $O \in K, R$ is a ternary accessibility relation on $K$ and ${ }^{*}$ - a unary operation on $K$. The following conditions for $R$ and ${ }^{*}$ are satisfied:

(p1) ROaa

(p2) Raaa

(p3) $R^{2} a b c d \Rightarrow R^{2} a c b d$

(p4) $R^{2} O a b c \Rightarrow R a b c$

(p5) $R a b c \Rightarrow R a c^{*} b^{*}$ 
(p6) $a^{* *}=a$

(p7) $R O a b \Rightarrow R O b a$

(q1) $R a b c \Rightarrow R b a c$

(d1) $a<b==_{\text {def }} R O a b$

(d2) $R^{2} a b c d={ }_{\text {def }} \exists x(R a b x \& R x c d \& x \in K)$.

A valuation $v$ is defined as a mapping assigning the truth value from truth-value matrix for $\mathrm{E} Q$ to propositional variables in every point of $K$ accounting the binary relation $<$ from (d1). An interpretation $I$ is a natural extending of $v$ on all formulas of ${\aleph_{\aleph_{0}}}_{\text {under }}$ condition that in any point of $K$ the usual explication of connectives takes place. The formal definition is as follows:

a) $v$ is a valuation in $\mathrm{L}$-frame, i.e. $v$ is a function $v: V \times K \rightarrow$ $M_{[0,1]}$ (where $M_{[0,1]}$ is a logical matrix for $\mathrm{E}_{\aleph_{0}}$ i.e. $M_{[0,1]}=$

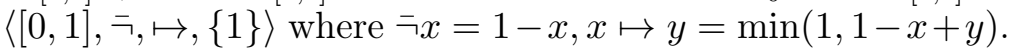
For any $p \in V$ and any $a, b \in K$ the following condition is satisfied:

(1) $a<b \& v(p, a) \neq 0 \Rightarrow(p, b) \neq 0$;

b) $I$ is an interpretation associated with $v$, i.e. $I$ is a function $I$ : $W^{0} \times K \rightarrow M_{[0,1]}$ satisfying for any $p \in V, A, B \in W^{0}, a \in K$ the following conditions:

(i) $I(p, a)=v(p, a)$;

(ii) $I\left(\neg A, a=1-x\right.$ iff $I\left(A, a^{*}\right)=x$;

(iii) $I(A \rightarrow B, a)=\inf (1,1-x+y)$ iff for any $b, c \in K$, Rabc and $I(a, b)=x \Rightarrow I(B, c)=y$.

(iv) $I(Q A, a)=1$ iff for any $b \in K(R O a b \Rightarrow \exists c \in K(R O b c \Rightarrow$ $I(A, c) \neq 0))$.

The following theorem was proved [8, p. 67]:

THEOREM 2. The system $E Q$ is complete in respect to the ternary semantic that is for any $A \in W^{0}$, if $I(A)=1$ for any intepretation $I$ then $\vdash$ A. 
We have the following finite model property.

The junction of both semantics of $\mathrm{€} Q$ can be achieved via putting for any $A \in W^{0}, \operatorname{dom}\left(I^{D}(A)\right) \subseteq K$ and $r n g\left(I^{D}(A)\right) \subseteq\{I(A, a)$ : $a \in K\}$, that is, treating the set $\Psi$ as $K$.

Proposition 1. A formula $F$ is valid in $E Q$ if and only if $F$ is valid in all those $E$-frames $\left\langle O, K, R,^{*}\right\rangle$ where $K$ is finite.

Proof. Let $\Pi=\left\langle O, K, R,{ }^{*}, I\right\rangle$ and let $V_{F}=\left\{p_{1}, \ldots, p_{n}\right\}$ be the propositional variables occurring in $F$. Moreover, let $\mathcal{B}_{F}$ be the set of all bi-valued assignments $I_{F}: V_{F} \rightarrow\{0,1\}$. We write $I_{F}^{a}$ if $\forall p \in$ $V: I_{F}(p)=I(p, a)$ and define a new model $\Pi_{f}=\left\langle O^{\prime}, K_{f}, R^{\prime},{ }^{* \prime}, I^{\prime}\right\rangle$ as follows:

- $K_{f}=\left\{I_{F} \in \mathcal{B}_{F}: \exists a \in K: I_{F}=I_{F}^{a}\right\}$

- $I^{\prime}\left(p, I_{F}\right)=I(p, a)$, where $I_{F}=I_{F}^{a}$

- $R^{\prime} \subseteq K_{f} \times K_{f} \times K_{f}$ where we take $R^{\prime} I(a) I(b) I(c)$ as corresponding to $R a b c$.

We can uniquely extend this to all subsets of $K_{f}$. It is straightforward to check that $I(F, O)=I^{\prime}\left(I_{F}^{O}, F\right)$. Thus we have shown that in evaluating $F$ it suffices to consider $\Pi_{f}$ with at most $2^{p(F)}$ where $p(F)$ is the number of different propositional variables occurring in $F$.

The analysis shows that we can replace the rule $(i v)$ with the rule $(i v)^{\prime}$ without the loss of the generality :

$(i v)^{\prime} I(Q A, a)=\inf \{I(A, c):$ for any $b \in K(R O a b \Rightarrow \exists c \in$ $K(R O b c \Rightarrow I(A, c) \neq 0\}$.

Turning back to the game theoretic semantic of $\mathrm{E}_{\aleph_{0}}$ it is worth to denote that recently some its extensions were obtained (cf. [2], [3]). It seems natural to adopt such an approach for producing this kind of semantics for $\mathrm{E} Q$ which is also, in fact, the specific extension of $\mathrm{E}_{\aleph_{0}}$. 


\section{Dialogue Game for $\mathbf{€} Q$}

In 2006 C. Fermúller and R. Kosik [2] proposed some game theoretic interpretation for the $S €$ system that extends both Lukasiewicz logic $\mathrm{E}_{\aleph_{0}}$ and modal logic $S 5$. It was builded on ideas already introduced by Robin Giles in the 1970th to obtain a characterization of $\mathrm{E}_{\aleph_{0}}$ in terms of a Lorenzen style dialogue game combined with bets on the results of binary experiments that may show dispersion. In [2] the experiments were replaced by random evaluations with respect to a given probability distribution over permissible precisifications. We will try to implement main ideas of interpretation proposed (respectively modifying it) for obtaining game theoretical semantic for the $\mathrm{E} Q$.

Assume that two players agree to pay $1 €$ to the opponent player for each assertion of an atomic statement, which is false in any $a \in K$ according to a randomly chosen set of observation points. More formally, given a set of all observation points $K$ the risk value $\langle x\rangle_{K}$ associated with a propositional variable $x$ is defined as $\langle x\rangle_{K}=$ $I^{D}(x)$. In fact, $\langle x\rangle_{K}$ corresponds to the probabilities of having to pay $1 €$, when asserting $x$.

Let $x_{1}, x_{2}, \ldots, y_{1}, y_{2} \ldots$ denote atomic statements, i.e. propositional variables. By $\left[x_{1}, \ldots, x_{m} \| y_{1}, \ldots, y_{n}\right]$ we denote an elementary state in the game where the 1st - the first player - asserts each of the $y_{i}$ in the multiset $\left\{y_{1}, \ldots, y_{n}\right\}$ of atomic statements and the 2 nd - the second player - asserts each atomic statement $x_{i} \in\left\{x_{1}, \ldots, x_{m}\right\}$. The risk associated with a multiset $X=\left\{x_{1}, \ldots, x_{m}\right\}$ of atomic formulas is defined as $\left\langle x_{1}, \ldots, x_{m}\right\rangle_{K}=\sum_{i=1}^{m}\left\langle x_{i}\right\rangle_{K}$. The risk \langle\rangle$_{K}$ associated with the empty multiset is $0 .\langle V\rangle_{K}$ respectively denotes the average amount of payoffs that the 1st player expects to have to pay to the 2nd player according to the above arrangements if he/she asserted the atomic formulas in $V$. The risk associated with an elementary state $\left[x_{1}, \ldots, x_{m} \| y_{1}, \ldots, y_{n}\right]$ is calculated from the point of view of the 1st player and therefore the condition $\left\langle x_{1}, \ldots, x_{m}\right\rangle_{K} \geq\left\langle y_{1}, \ldots, y_{n}\right\rangle_{K}$ (success condition) expresses that the 1st player does not expect any loss (but possibly some gain) when betting on the truth of atomic statements.

Now we accept the following dialogue rule for implication (cf. [2]): 
$\left(R_{\rightarrow}\right)$ If the 1st player asserts $A \rightarrow B$ in point $a$ then, whenever the 2nd player chooses to attack this statement by asserting $A$ in point $b$, the 1 st has to assert also $B$ in point $c$ (the points are choosing according to the condition (iii) above). (And vice versa, i.e., for the roles of 1 st and the 2 nd player switched.)

A player may also choose not to attack the opponent's assertions of $A \rightarrow B$. The rule reflects the idea that the meaning of implication entails the principle that an assertion of 'If $A$ then $B$ ' obliges one to assert also $B$ if the opponent in a dialogue grants (i.e. asserts) $A$.

The dialogue rule for the negation involves a relativization to specific observation points:

$(R \neg)$ If the 1st player asserts $\neg A$ in point $a$ then the 2 nd player chooses to attack this statement by asserting $A$ in point $a^{*}$ (the points are choosing according to the condition (iii) above). (And vice versa, i.e., for the roles of 1 st and the 2 nd player switched.)

The dialogue rule for the $Q$-modality also involves a relativization to specific observation points:

$(R Q)$ If the 1st player asserts $Q A$ then the 1st also have to assert that $A$ holds (its interpretation differs from 0 ) at any point that the 2nd may choose using the condition (iv) above (And vice versa, i.e., for the roles of the 1 st and the 2 nd switched.)

Henceforth we will use $A^{a}$ as shorthand for ' $A$ holds at the observation point $a^{\prime}$ and speak of $A$ as a formula indexed by $a$, accordingly. Thus using rule $(R Q)$ entails that we have to deal with indexed formulas also in rule $\left(R_{\rightarrow}\right)$. However, we don't have to change the rule itself, which will turn out to be adequate independently of the kind of evaluation that we aim at in a particular context. We only need to stipulate that in applying $\left(R_{\rightarrow}\right)$ the observation point index of $A \rightarrow B$ (if there is any) is used for defininig the respective indexes for the subformulas $A$ and $B$. If, on the other hand, we apply rule $(R Q)$ to an already indexed formula $(Q A)^{a}$ then the in$\operatorname{dex} a$ is overwritten by whatever index $b$ is chosen by the opponent 
player; i.e., we have to continue with the assertion $A^{b}$ and, of course, we also have to account for indices of formulas in elementary states. We augment the definition of risk by $\left\langle x^{a}\right\rangle_{K}=1-I(x, a)$. In other words, the probability of having to pay $1 €$ for claiming that $x$ holds at the observation point $a$ is 0 if $x$ is true at $a$ and 1 if $x$ is false at $a$.

We use $\left[A_{1}^{a_{1}}, \ldots, A_{m}^{a_{m}} \| B_{1}^{b_{1}}, \ldots, B_{n}^{b_{n}}\right]$ to denote an arbitrary (not necessarily elementary) state of the game, where $\left\{A_{1}^{a_{1}}, \ldots, A_{m}^{a_{m}}\right\}$ is the multiset of formulas that are currently asserted by the 2 nd player, and $\left\{B_{1}^{b_{1}}, \ldots, B_{n}^{b_{n}}\right\}$ is the multiset of formulas that are currently asserted by the 1st player. (We don't care about the order in which formulas are asserted.)

A move initiated by the1st player (1st-move) in state $[\Gamma|| \Delta]$ consists in his/her picking of some non-atomic formula $A^{a}$ from the multiset $\Gamma$ and proceeding as follows:

- If $A^{a}=\left(A_{1} \rightarrow A_{2}\right)^{a}$ then the 1st may either attack by asserting $A_{1}^{b}$ in order to force the 2 nd to assert $A_{2}^{c}$ in accordance with $\left(R_{\rightarrow}\right)$, or admit $A^{a}$. In the first case the successor state is $\left[\Gamma^{\prime}, A_{2}^{c} \| \Delta, A_{1}^{b}\right]$, in the second case it is $\left[\Gamma^{\prime} \| \Delta\right]$, where $\Gamma^{\prime}=$ $\Gamma-\left\{A^{a}\right\}$.

- If $A^{a}=\left(\neg A_{1}\right)^{a}$ then the 1st chooses the point $a^{*}$ thus forcing the 2 nd to assert $A_{1}^{a^{*}}$. The successor state is $\left[\Gamma, A_{1}^{a^{*}} \| \Delta^{\prime}\right]$, where $\Delta^{\prime}=\Delta-\left\{A^{a}\right\}$.

- If $A^{a}=Q B^{a}$ then the 1st chooses an arbitrary $b \in K$ using the condition $(i v)$ above thus forcing the 2nd to assert $B^{c}$. The successor state is $\left[\Gamma^{\prime}, B^{c} \| \Delta\right]$, where $\Gamma^{\prime}=\Gamma-\left\{A^{a}\right\}$.

A move initiated by the 2 nd player (2-move) is symmetric, i.e. with the roles of the 1st and the 2nd players interchanged. A run of the game consists in a sequence of states, each resulting from a move in the immediately preceding state, and ending in an elementary state $\left[x_{1}^{a_{1}}, \ldots, x_{m}^{a_{m}} \| y_{1}^{b_{1}}, \ldots, y_{n}^{b_{n}}\right]$. The 1st player succeeds in this run if this final state fulfills the success condition, i.e., if

$$
\sum_{j=1}^{n}\left\langle y_{j}^{b_{j}}\right\rangle_{K}-\sum_{i=1}^{m}\left\langle x_{i}^{a_{i}}\right\rangle_{K} \leq 0 .
$$


The term at the left hand side of inequality is an expected loss of the 1st player at this state. In other words, the 1st succeeds if its expected loss is 0 or even negative, i.e., in fact a gain. The other connectives can be reduced to implication and negation.

\section{Adequacy of the game}

To show that the considered game indeed characterizes logic $\mathrm{\iota} Q$, we have to analyse all possible runs of the game starting with some arbitrarily complex assertion by the 1st player. A strategy for the 1st player will be a tree-like structure, where a branch represents a possible run resulting from particular choices made by the 1st player, taking into account all possible choices of the 2nd player in (2- or 1-moves) that are compatible with the rules. We will only have to look at strategies for the 2nd player and thus call a strategy winning if the 1st player succeeds in all corresponding runs (according to condition (2)).

Taking into account that by Theorem (2) we can assume that the set $K$ of observation points (states) is finite. The construction of strategies can be viewed as systematic proof search in an analytic tableau calculus with the following rules:

$$
\begin{aligned}
& \frac{\left[\Gamma \| \Delta,\left(A_{1} \rightarrow A_{2}\right)^{a}\right]}{\left[\Gamma, A_{1}^{b} \| \Delta, A_{2}^{c}\right] \mid[\Gamma \| \Delta]}\left(\rightarrow_{2 n d}\right) \\
& \frac{\left[\Gamma,\left(A_{1} \rightarrow A_{2}\right)^{a}|| \Delta\right]}{\left[\Gamma, A_{2}^{c}|| \Delta, A_{1}^{b}\right]}\left(\rightarrow_{1 s t}^{1}\right) \quad \frac{\left[\Gamma,\left(A_{1} \rightarrow A_{2}\right)^{a}|| \Delta\right]}{[\Gamma|| \Delta]}\left(\rightarrow_{1 s t}^{2}\right) \\
& \frac{\left[\Gamma \| \Delta,(\neg A)^{a}\right]}{\left[\Gamma, A^{a^{*}} \| \Delta\right]}(\neg 2 n d) \quad \frac{\left[\Gamma,(\neg A)^{a} \| \Delta\right]}{\left[\Gamma \| \Delta, A^{a^{*}}\right]}(\neg 1 s t) \\
& \frac{\left[\Gamma \| \Delta,(Q A)^{a}\right]}{\left[\Gamma|| \Delta, A^{c_{1}}\right]|\ldots|\left[\Gamma|| \Delta, A^{\left.c_{n}\right]}\right.}\left(Q_{2 n d}\right) \frac{\left[\Gamma,(Q A)^{a} \| \Delta\right]}{\left[\Gamma, A^{c}|| \Delta\right]}\left(Q_{1 s t}\right)
\end{aligned}
$$

In all rules $a$ can denote any index. In the rule $\left(Q_{2 n d}\right)$ as well as in the rule $\left(Q_{1 s t}\right)$ we assume that indexes $c_{1}, \ldots, c_{n}$ and $c$ are defined by means of the condition ( $i v)$ above. Note that, in accordance with the definition of a strategy for the 2nd player, his/her choices in the moves induce branching, whereas for the 1st player choices a single successor state that is compatible with the dialogue rules is chosen. 
TheOREM 3. A formula $F$ is valid in $E Q$ if and only if for every set $K$ of observation points (states) the 1st player have a winning strategy for the game starting in game state $[\| F]$.

Proof. Every run of the game is finite. For every final elementary state $\left[x_{1}^{a_{1}}, \ldots, x_{m}^{a_{m}} \| y_{1}^{b_{1}}, \ldots, y_{n}^{b_{n}}\right]$ the success condition says that we have to compute the risk $\sum_{j=1}^{n}\left\langle y_{j}^{b_{j}}\right\rangle_{K}-\sum_{i=1}^{m}\left\langle x_{i}^{a_{i}}\right\rangle_{K}$, where $\left\langle r^{a}\right\rangle_{K}=$ $I(r, a)$ if $a \notin \operatorname{dom}\left(I^{D}(r)\right)$ and $\left\langle r^{a}\right\rangle_{K}=1-I^{D}(r)(a)$ otherwise, and check whether the resulting value (in the following denoted by $\left.\left\langle x_{1}^{a_{1}}, \ldots, x_{m}^{a_{m}} \| y_{1}^{b_{1}}, \ldots, y_{n}^{b_{n}}\right\rangle\right)$ is $\leq 0$ to determine whether the 1st player 'win' the game. To obtain the minimal final risk of the 1st player (i.e., his/her minimal expected loss) that the 1st can enforce in any given state $S$ by playing according to an optimal strategy, we have to take into account the supremum over all risks associated with the successor states to $S$ that you can enforce by a choice that you may have in a (2nd- or 1st-)move $S$. On the other hand, for any of the 1st player choices the 1st can enforce the infimum of risks of corresponding successor states. In other words, we prove that we can extend the definition of the 1st expected loss from elementary states to arbitrary states such that the following conditions are satisfied:

(3.1) $\left\langle\Gamma,(A \rightarrow B)^{a}|| \Delta\right\rangle_{K}=\inf \left\{\langle\Gamma|| \Delta\rangle_{K},\left\langle\Gamma, B^{c} \| A^{b}, \Delta\right\rangle_{K}\right\}$

$(3.2)\left\langle\Gamma,(\neg A)^{a}|| \Delta\right\rangle_{K}=\sup \left\{\left\langle\Gamma|| \Delta, A^{a^{*}}\right\rangle_{K}\right\}$

for assertions by the 2nd player and, for assertions by the 1st player:

$$
\begin{aligned}
& \left\langle\Gamma \|(A \rightarrow B)^{a}, \Delta\right\rangle_{K}=\sup \left\{\left\langle\Gamma, A^{b} \| B^{c}, \Delta\right\rangle_{K},\langle\Gamma \| \Delta\rangle_{K}\right\} \\
& \left.\left\langle\Gamma \| \Delta,(\neg A)^{a}\right\rangle_{K}=\inf \left\{\left\langle\Gamma, A^{a^{*}} \| \Delta\right\rangle_{K}\right\rangle\right\}
\end{aligned}
$$

Furthermore we have

$$
\begin{aligned}
\left\langle\Gamma|| \Delta,(Q A)^{a}\right\rangle_{K} & =\sup _{\substack{c \in K \\
R O a b \Rightarrow R O b c}}\left\{\left\langle\Gamma|| \Delta, A^{c}\right\rangle_{K}\right\} \\
\left\langle\Gamma,(Q A)^{a}|| \Delta\right\rangle_{K}= & \inf _{\substack{c \in K \\
R O a b \Rightarrow R O b c}}\left\{\left\langle\Gamma, A^{c} \| \Delta\right\rangle_{K}\right\}
\end{aligned}
$$


We have to check that $\langle.|| .\rangle_{K}$ is well-defined; i.e., that conditions above together with the definition of my expected loss (risk) for elementary states indeed can be simultaneously fulfilled and guarantee uniqueness. To this aim consider the following generalisation of the truth function for $\mathrm{€} Q$ to multisets $G$ of indexed formulas:

$$
I(\Gamma)_{K}=\operatorname{def}_{\substack{A \in \Gamma \\ a \notin \operatorname{dom}\left(I^{D}(A)\right)}} I(A, a)+\sum_{\substack{A \in \Gamma \\ a \in \operatorname{dom}\left(I^{D}(A)\right)}} I^{D}(A)(a)
$$

Note that

$$
I(\{A\})_{K}=I(A)_{K}=\sum_{a \notin \operatorname{dom}\left(I^{D}(A)\right)} I(A, a)+\sum_{a \in \operatorname{dom}\left(I^{D}(A)\right)} I^{D}(A)(a)=1 \text { iff }\langle\| A\rangle_{K} \leq 0,
$$

that is, $A$ is valid in $\mathrm{E} Q$ iff my risk in the game starting with my assertion of $A$ is non-positive. Moreover, for elementary states we have

$\left\langle x_{1}^{a_{1}}, \ldots, x_{m}^{a_{m}} \| y_{1}^{b_{1}}, \ldots, y_{n}^{b_{n}}\right\rangle_{K}=n-m+I\left(x_{1}^{a_{1}}, \ldots, x_{m}^{a_{m}}\right)_{K}-I\left(y_{1}^{b_{1}}, \ldots\right.$, $\left.y_{n}^{b_{n}}\right)_{K}$.

We generalize the risk function to arbitrary observation states by

$$
\langle\Gamma|| \Delta\rangle_{K}^{*}{ }_{\text {def }}|\Delta|-|\Gamma|+I(\Gamma)_{K}-I(\Delta)_{K}
$$

and check that it satisfies conditions (3.1)-(3.6). We only spell out two cases. In order to avoid case distinctions let $I\left(A^{a}\right)_{K}=I(A, a)$. For condition (3.1) we have

$\left\langle\Gamma,(A \rightarrow B)^{a}|| \Delta\right\rangle_{K}^{*}=|\Delta|-|\Gamma|-1+I(\Gamma)_{K}+I(A \rightarrow B, a)_{K}-$ $I(\Delta)_{K}=\langle\Gamma|| \Delta\rangle_{K}^{*}-1+I(A \rightarrow B, a)=\langle\Gamma|| \Delta\rangle_{K}^{*}-1+\inf \{1,1-$ $I(A, b)+I(B, c)\}=\langle\Gamma|| \Delta\rangle_{K}^{*}-1+\inf \left\{1,1+\left\langle B^{c}|| A^{b}\right\rangle_{K}^{*}\right\}=\langle\Gamma|| \Delta\rangle_{K}^{*}+$ $\inf \left\{0,\left\langle B^{c} \| A^{b}\right\rangle_{K}^{*}\right\}=\inf \left\{\langle\Gamma|| \Delta\rangle_{K}^{*},\left\langle\Gamma, B^{c} \| A^{b}, \Delta\right\rangle_{K}^{*}\right\}$.

For condition (3.5) we have

$\left\langle\Gamma|| \Delta,(Q A)^{a}\right\rangle_{K}^{*}=|\Delta|-|\Gamma|-1+I(\Gamma)_{K}-I(\Delta)_{K}-I\left((Q A)^{a}\right)_{K}=$ $\langle\Gamma|| \Delta\rangle_{K}^{*}+1-I(Q A, a)=\langle\Gamma|| \Delta\rangle_{K}^{*}+1-\inf \{I(A, c)$ : for any $b \in K\left(R O a b \Rightarrow \exists c \in K(R O b c \Rightarrow I(A, c) \neq 0\}=\langle\Gamma|| \Delta\rangle_{K}^{*}+\right.$ 
$\sup \{I(A, c)$ :for any $b \in K(R O a b \Rightarrow \exists c \in K(R O b c \Rightarrow I(A, c) \neq 0\}=$ $\sup _{\substack{c \in K \\ R O a b \Rightarrow R O b c}}\left\{\left\langle\Gamma \| \Delta, A^{c}\right\rangle_{K}^{*}\right\}$

Let us define a regulation as assignment of labels 'the 2nd player moves next' and 'the 1st player moves next' to game states that obviously constrain the possible runs of the game. A regulation is consistent if the label ' $2 n d($ Ist $)$ move next' is only assigned to states where such a move is possible, i.e., where 1st player (2nd player) have asserted a non-atomic formula. As a corollary to our proof of Theorem (3), we obtain:

COROllary 1. The total expected loss $\langle\Gamma|| \Delta\rangle_{K}^{*}$ that the 1 st player can enforce in a game over $K$ starting in state $[\Gamma \| \Delta]$ only depends on $\Gamma, \Delta$ and $K$. In particular, it is the same for every consistent regulation that may be imposed on the game.

\section{References}

[1] Dishkant, H., An Extension of the Eukasiewicz Logic to the Modal Logic of Quantum Mechanics, Studia Logica 37(2):149-155, 1976.

[2] Fermüller, C. G., and R. KosiK, Combining supervaluation and degree based reasoning under vagueness, Proceedings of LPAR 2006, volume 4246 of LNAI. Springer, 2006, pp. 212-226.

[3] Fermüller, C. G., Revisiting Giles - connecting bets, dialogue games, and fuzzy logics, in O. Majer, A.V.Pietarinen and T. Tulenheimo (eds.), Games: Unifying Logic, Language, and Philosophy, Springer, 2009, pp. 209-227.

[4] Giles, R., A non-classical logic for physics, Studia Logica 33(4):399417, 1974.

[5] Giles, R., A non-classical logic for physics, in R. Wojcicki, G. Malinowski (eds.), Selected Papers on Eukasiewicz Sentential Calculi, Polish Academy of Sciences, 1977, pp. 13-51.

[6] Mackey, G. W., The Mathematical Foundations of Quantum Mechanics, New York, 1963.

[7] Vasyukov, V. L., The Completeness of the Factor Semantics for Łukasiewicz's Infinite-valued Logics, Studia Logica 52(1):143-167, 1993.

[8] Vasyukov, V. L., Quantum Logics, Moscow: Per Se, 2005 (in Russian). 